\section{A insercão da subárea de Atividade Física e Saúde nos programas de pós-graduação em Educação Física no Brasil}

\author{
Insertion of the Health-Related Physical \\ Activity field in the Physical Education \\ postgraduate courses in Brazil
}

Leandro Martin Totaro Garcia'

Andrea Wendt Böhm²

Eliane Denise Araújo Bacil ${ }^{3}$

Maurício Feijó da $\mathrm{Cruz}^{2}$

Rafaela Cavalheiro do Espírito Santo

\section{RESUMO}

O objetivo deste trabalho foi verificar a inserção da subárea de Atividade Física e Saúde (AFS) nos programas de pós-graduação (PPG) em Educação Física no Brasil. Trata-se de estudo descritivo baseado em análise de dados secundários, presentes nos relatórios da avaliação de 2012 da Coordenação de Aperfeiçoamento de Pessoal de Nível Superior (CAPES), realizado em março de 2014. Foram selecionados os PPG que ofertavam mestrado ou doutorado acadêmico em Educação Física. Quantificaram-se as áreas de concentração, linhas de pesquisa, projetos de pesquisa em andamento, docentes permanentes e colaboradores e alunos totais em cada PPG, assim como aquelas pertencentes à subárea de AFS. Na análise dos dados se utilizou a estatística descritiva, estratificações por macrorregiões geográficas e conceitos na avaliação da CAPES. Foram avaliados 26 PPG, dos quais três não apresentaram área de concentração que contemplava a AFS. Do total, 55,8\% das áreas de concentração, 25,0\% das linhas de pesquisa, 26,0\% dos projetos de pesquisa, 38,7\% dos professores e $29,7 \%$ dos alunos têm vinculação com a AFS. Os PPG com conceitos 5 e 3 apresentaram maior contribuição da AFS, enquanto os PPG com conceito 6 apresentaram menores proporções. Os padrões de inserção da AFS nas macrorregiões foram díspares. Conclui-se que a subárea de AFS tem posição de destaque nos PPG em Educação Física no Brasil. No entanto, desequilíbrios regionais e entre instituições mais e menos consolidadas indicam desafios para o futuro.

\section{PALAVRAS-CHAVE}

Indicadores de projetos de pesquisa e desenvolvimento; Indicadores de recursos humanos em ciência e tecnologia; Programas de pós-graduação em saúde; Atividade motora.

\begin{abstract}
The goal of this study was to verify the insertion of the Health-Related Physical Activity (HRPA) field in Physical Education (PE) postgraduate courses (PGC) in Brazil. This is a descriptive study based on analysis of secondary data found in reports of the 2012 evaluation of the Coordination of Improvement of Higher Education Personnel (CAPES), held in March 2014. PGC that offer academic masters or doctoral degree in PE were selected. The total concentration areas, research $l i-$ nes, research projects ongoing, permanent and collaborators faculty, and students in each PGC were quantified, as well as those belonging to the HRPA field. Analysis of data was descriptively, stratified by geographic macro-regions and by the grades in the CAPES evaluation. Were evaluated 26 courses, of which three showed no concentration area that included HRPA. Of all, $55.8 \%$ of the concentration areas, $25.0 \%$ of the research lines, $26.0 \%$ of the projects, $38.7 \%$ of the faculty, and $29.7 \%$ of the students have linkage with HRPA. PGC with grades 5 and 3 showed higher contribution of HRPA. However, PGC with grade 6 showed the lowest proportions of contribution of HRPA. The patterns of insertion of the HRPA field in the macro-regions were disparate. It is concluded that the HRPA field has a prominent position in Physical Education PGC in Brazil. Nevertheless, regional and institutional imbalances indicate challenges for the future.
\end{abstract}

\section{KEYWORDS}

Research and development project indicators; Human resources in science and technology indicators; Health postgraduate programs; Motor activity.
Rev Bras Ativ Fis Saúde p. 215-222 $\mathrm{DOI}$

http://dx.doi.org/10.12820/rbafs.v.19n2p215

1 Programa de Pós-Graduação em Nutricão em Saúde Pública, Universidade de São Paulo, São Paulo, Brasil.

2 Programa de Pós-Graduação em Epidemiologia, Universidade Federal de Pelotas, Pelotas, Brasil.

3 Programa de Pós-Graduação em Educação Física, Universidade Federal do Paraná, Curitiba, Brasil.

4 Programa de Pós-Graduação em Ciências Médicas, Universidade Federal do Rio Grande do Sul, Porto Alegre, Brasil. 


\section{INTRODUÇÃO}

Com a valorização da área acadêmica no Brasil, houve um aumento da quantidade de pesquisadores em Educação Física (EF) e da representatividade deles na grande área da saúde, reflexo das políticas de incentivo à pós-graduação em EF implementadas a partir da década de $1970^{1,2}$.

Dentro dos programas de pós-graduação (PPG) da EF observa-se uma crescente evolução numérica e qualitativa em pesquisas na subárea "Atividade Física e Saúde" (AFS) ${ }^{1,3,4}$, a qual vem ganhando espaço em diversos centros de ensino, devido ao interesse de pesquisadores e profissionais em investigar esse tópico. Isso em parte reflete a presença da prática de atividade física como uma das prioridades em saúde pública e em debates acadêmicos da área de saúde 5 .

Por ser uma subárea menos tradicional ${ }^{1}$, quantificar a inserção dela dentro da EF é de suma importância, pois permite identificar o seu espaço como campo de conhecimento, a existência de desequilíbrios regionais, almejar maior reconhecimento das agências de fomento e da comunidade científica e estabelecer metas mais ousadas para si.

Sendo assim, o objetivo do presente estudo foi verificar a inserção da subárea de AFS nos PPG em EF no Brasil.

\section{MÉTODOS}

Trata-se de um estudo descritivo baseado em análise secundária de dados, em que se utilizaram os relatórios da avaliação do ano de 2012 da Coordenação de Aperfeiçoamento de Pessoal de Nível Superior (CAPES), uma fundação do Ministério da Educação do Brasil. As informações foram obtidas a partir dos documentos disponíveis em repositórios de acesso público do sítio eletrônico da CAPES ${ }^{6}$. No momento da coleta de dados, a última atualização do sítio eletrônico havia ocorrido em 11 de março de 2014.

Foram selecionados para o estudo os PPG que constavam na relação de cursos recomendados e reconhecidos pela CAPES na área de EF e que ofertavam mestrado ou doutorado acadêmico. Para caracterizar os PPG, foram coletados do sítio eletrônico da CAPES o nome da Instituição de Ensino Superior (IES) de origem, a macrorregião geográfica (Norte; Nordeste; Centro -Oeste; Sudeste; Sul), os níveis ofertados (Mestrado; Mestrado e Doutorado) e os conceitos na avaliação da CAPES $(3 ; 4 ; 5 ; 6)$.

A partir dos documentos "Proposta do Programa" de cada PPG, foram quantificadas as áreas de concentração e as linhas de pesquisa. Em seguida, os nomes e as descrições de todas as linhas de pesquisa foram lidos dos documentos "Linha de Pesquisa" por duas pessoas independentemente, a fim de selecionar as linhas vinculadas à subárea de AFS. Foram selecionadas as linhas que: a) continham o termo "Saúde" e "Atividade Física" ou "Exercício Físico" ou "Educação Física" no nome; ou b) apresentavam na descrição aproximação epistemológica com a subárea, utilizando como critério o pertencimento aos paradigmas apresentados por $\mathrm{Loch}^{4}$, a saber: paradigma centrado na aptidão física e no exercício físico e paradigma centrado na atividade física. Quando houve discordância entre os dois avaliadores, tentou-se primeiramente o consenso, e quando não foi possível, um terceiro avaliador foi chamado. Definiu-se como área de concentração vinculada à AFS aquela que apresentou ao 
menos uma linha de pesquisa vinculada à subárea.

Identificou-se o total de docentes permanentes e colaboradores em cada PPG nos documentos "Formação, Vinculação e Área de Concentração do Docente". Em seguida, a partir do documento "Relação Nominal de Docentes Responsáveis" se verificou a quantidade de docentes dos PPG responsáveis ou colaboradores em projetos de pesquisa vinculados às linhas de pesquisa identificadas anteriormente como pertencentes à AFS, ou que orientavam dissertações e teses nessas linhas. Por fim, nos documentos "Projetos de pesquisa em andamento, financiados e com participação de discentes" se obteve a quantidade total de projetos de pesquisa em andamento em cada PPG e de alunos de mestrado e doutorado vinculados a eles, assim como daqueles projetos vinculados às linhas de pesquisa definidas como pertencentes à subárea de AFS.

Para a análise dos dados foi utilizada a estatística descritiva (frequências absolutas e relativas), a fim de identificar a proporção de áreas de concentração, linhas de pesquisa, projetos de pesquisa em andamento, docentes e alunos vinculados à subárea de $\mathrm{AFS}$ nos $\mathrm{PPG}$ da área da $\mathrm{EF}$. Realizaram-se também análises estratificadas por macrorregião geográfica e conceito na avaliação da CAPES. As análises foram conduzidas no software Microsoft ${ }^{\circledR}$ Office ${ }^{\circledR}$ Excel 2010, versão para Microsoft ${ }^{\circledR}$ Windows $^{\mathrm{TM}}$.

\section{RESULTADOS}

Dos 32 PPG relatados pela CAPES na relação de cursos recomendados e reconhecidos na área de EF, 26 (81,3\%) foram analisados. Dos seis excluídos, dois não constavam como homologados na data de coleta (Ciências da Atividade Física - USP, e Ciências do Movimento Humano - UNIMEP), um ofertava somente mestrado profissionalizante (Exercicio Fisico na Promoção da Saúde - UNOPAR) e três apresentavam baixa relação epistemológica (Fonoaudilogia - UNESP/MAR, Reabilitação e Desempenho Funcional - USP/ RP, e Terapia Ocupacional - UFSCAR).

Observou-se que a região com maior quantidade de PPG na área de EF é a Sudeste, com 13 PPG, seguida da região Sul $(n=7)$ e regiões Nordeste e Centro-Oeste (n=3, cada uma). Dentre todos os PPG na EF, três não apresentavam área de concentração que contemplava AFS. Observando a inserção da subárea de AFS, percebeu-se que 55,8\% das áreas de concentração, 25,0\% das linhas de pesquisa e 26,0\% dos projetos de pesquisa dos PPG eram pertencentes à AFS. Além disso, 38,7\% dos professores e 29,7\% dos alunos dos PPG estavam envolvidos na subárea de AFS (Tabela 1).

$\mathrm{Na}$ Tabela 1 observa-se a distribuição das IES de acordo com o conceito da avaliação da CAPES, apresentando as proporções dos PPG que têm áreas de concentração, linhas de pesquisa, projetos de pesquisa em andamento, professores e alunos pertencentes à subárea AFS. Os programas que receberam conceitos 5 e 3 foram os que apresentaram maior contribuição da AFS. Os PPG com conceito 5 apresentaram as maiores proporções de áreas de concentração, linhas de pesquisa, docentes e alunos em AFS. No entanto, os PPG com melhor avaliação da CAPES apresentaram as menores proporções de contribuição da AFS para todos os itens. 
TABELA 1 - Atividade Física e Saúde em áreas de concentração, linhas de pesquisa, projetos de pesquisa em andamento, docentes e alunos em Programas de Pós-Graduação em Educação Física avaliados pela Área 21 da CAPES, em 2012.

\begin{tabular}{|c|c|c|c|c|c|c|c|}
\hline \multirow{2}{*}{ Conceitos/IES } & \multirow{2}{*}{ Região } & \multirow{2}{*}{ Nível } & Áreas & Linhas & Projetos & Docentes & Alunos \\
\hline & & & $N(\%)$ & $N(\%)$ & $\mathrm{N}(\%)$ & $\mathrm{N}(\%)$ & $\mathrm{N}(\%)$ \\
\hline \multicolumn{8}{|l|}{6} \\
\hline UNESP/RC & SE & $M / D$ & $1(33,3)$ & $2(22,2)$ & $17(23,0)$ & $10(41,7)$ & $84(23,6)$ \\
\hline USP & SE & $M / D$ & $1(33,3)$ & $1(9,1)$ & $5(3,1)$ & $3(6,1)$ & $4(2,4)$ \\
\hline Total do conceito & & & $2(33,3)$ & $3(15,0)$ & $22(9,3)$ & $13(17,8)$ & $88(16,9)$ \\
\hline \multicolumn{8}{|l|}{5} \\
\hline UFPR & S & $M / D$ & $1(100)$ & $1(33,3)$ & $21(42,0)$ & $13(59,1)$ & $84(48,8)$ \\
\hline UFRGS & S & $M / D$ & $1(50,0)$ & $1(25,0)$ & $27(22,7)$ & $11(40,7)$ & $44(21,5)$ \\
\hline UFSC & S & $M / D$ & $1(33,3)$ & $2(33,3)$ & $34(38,2)$ & $13(52,0)$ & $63(41,4)$ \\
\hline Total do conceito & & & $3(100)$ & $4(30,8)$ & $82(31,8)$ & $37(50,0)$ & $191(36,1)$ \\
\hline \multicolumn{8}{|l|}{4} \\
\hline UCB & $\mathrm{CO}$ & $M / D$ & $1(100)$ & $3(75,0)$ & $25(59,5)$ & $15(93,7)$ & $77(84,6)$ \\
\hline UEL & S & $M / D$ & $1(50,0)$ & $1(20,0)$ & $12(20,3)$ & $12(38,7)$ & $30(25,0)$ \\
\hline UFMG & SE & $M / D$ & - & - & - & - & - \\
\hline UGF & SE & $M / D$ & $1(50,0)$ & $1(25,0)$ & $11(32,4)$ & $9(52,9)$ & $21(28,8)$ \\
\hline UNICAMP & SE & $M / D$ & $1(33,3)$ & $1(14,3)$ & $10(13,9)$ & $9(23,1)$ & $18(17,1)$ \\
\hline UNICSUL & SE & $M / D$ & $1(100)$ & $1(25,0)$ & $2(20,0)$ & $6(54,5)$ & $11(27,5)$ \\
\hline UNIMEP & SE & M & - & - & - & - & - \\
\hline USJT & SE & $M / D$ & $1(100)$ & $1(25,0)$ & $21(36,8)$ & $7(50,0)$ & $33(35,9)$ \\
\hline Total do conceito & & & $6(50,0)$ & $8(22,9)$ & $81(23,1)$ & $58(38,2)$ & $190(29,1)$ \\
\hline \multicolumn{8}{|l|}{3} \\
\hline FESP/UPE & NE & M & $1(50,0)$ & $2(40,0)$ & $20(46,5)$ & $11(57,9)$ & $38(53,5)$ \\
\hline FUFSE & NE & M & $1(100)$ & $1(33,3)$ & $7(24,1)$ & $6(54,5)$ & $2(33,3)$ \\
\hline UFRN & NE & M & $1(50,0)$ & $1(33,3)$ & $17(50,0)$ & $6(60,0)$ & $8(40,0)$ \\
\hline UFMT & $\mathrm{CO}$ & M & $1(100)$ & $1(33,3)$ & $6(25,0)$ & $5(50,0)$ & $3(60,0)$ \\
\hline UNB & $\mathrm{CO}$ & M & $1(100)$ & $1(33,3)$ & - & - & - \\
\hline UDESC & S & $M / D$ & $1(100)$ & $1(33,3)$ & $45(48,9)$ & $14(63,6)$ & $84(50,6)$ \\
\hline UFPEL & S & M & $2(66,7)$ & $2(22,2)$ & $26(44,8)$ & $13(61,9)$ & $34(43,0)$ \\
\hline UFSM & S & M & $1(100)$ & $1(50,0)$ & $12(60,0)$ & $6(54,5)$ & $4(66,7)$ \\
\hline UFES & SE & M & $1(50,0)$ & $1(16,7)$ & $3(5,4)$ & $6(27,3)$ & $3(5,9)$ \\
\hline UFRJ & SE & M & - & - & - & - & - \\
\hline UFTM & SE & M & $1(100)$ & $1(33,3)$ & $8(23,5)$ & $5(33,3)$ & $15(34,9)$ \\
\hline UFV & SE & M & $1(50,0)$ & $1(33,3)$ & $5(25,0)$ & $7(53,8)$ & $19(25,0)$ \\
\hline UNIVERSO & SE & $M$ & $1(100)$ & $1(50,0)$ & $12(41,4)$ & $5(35,7)$ & $18(39,1)$ \\
\hline Total do conceito & & & $13(68,4)$ & $14(29,2)$ & $84(42,6)$ & $228(35,6)$ & $161(33,1)$ \\
\hline Total geral & & & $24(55,8)$ & $29(25,0)$ & $346(26,0)$ & $192(38,7)$ & $697(29,7)$ \\
\hline
\end{tabular}

CAPES: Coordenação de Aperfeiçoamento de Pessoal de Nível Superior. IES: Instituição de Ensino Superior. S: Sul; SE: Sudeste; CO: Centro-Oeste; NE: Nordeste. M: mestrado; M/D: mestrado e doutorado. Total do conceito: valores considerando todas as IES com o mesmo conceito na avaliação da CAPES em 2012. Total geral: valores para a totalidade das IES. UNESP/RC: Universidade Estadual Paulista Júlio de Mesquita Filho, campus Rio Claro; USP: Universidade de São Paulo; UFPR: Universidade Federal do Paraná; UFRGS: Universidade Federal do Rio Grande do Sul; UFSC: Universidade Federal de Santa Catarina; UCB: Universidade Católica de Brasília; UEL: Universidade Estadual de Londrina; UFMG: Universidade Federal de Minas Gerais; UGF: Universidade Gama Filho; UNICAMP: Univerisade Estadual de Campinas; UNICSUL: Universidade Cruzeiro do Sul; UNIMEP: Universidade Metodista de Piracicaba; USJT: Universidade São Judas Tadeu; FESP/UPE: Fundação Universidade de Pernambuco; FUFSE: Fundação Universidade Federal de 
Sergipe; UFRN: Universidade Federal do Rio Grande do Norte; UFMT: Universidade Federal do Mato Grosso; UNB:Universidade de Brasília; UDESC: Universidade do Estado de Santa Catarina; UFPEL: Universidade Federal de Pelotas; UFSM: Universidade Federal de Santa Maria; UFES: Universidade Federal do Espírito Santo; UFRJ: Universidade Federal do Rio de Janeiro; UFTM: Universidade Federal do Triângulo Mineiro; UFV: Universidade Federal de Viçosa; UNIVERSO: Universidade Salgado de Oliveira.

Verificou-se que os padrões de inserção de áreas de concentração, linhas de pesquisa, projetos, docentes e alunos vinculados à AFS nas macrorregiões foram, em geral, semelhantes. No entanto, a região Norte não apresentava PPG na área de EF. Todos os PPG da área de EF da região Centro-Oeste apresentavam área de concentração em AFS e 50\% das linhas de pesquisa eram relacionadas a ela. A região Nordeste apresentou as maiores proporções para docentes, projetos de pesquisa e alunos vinculados à AFS (Figura 1).

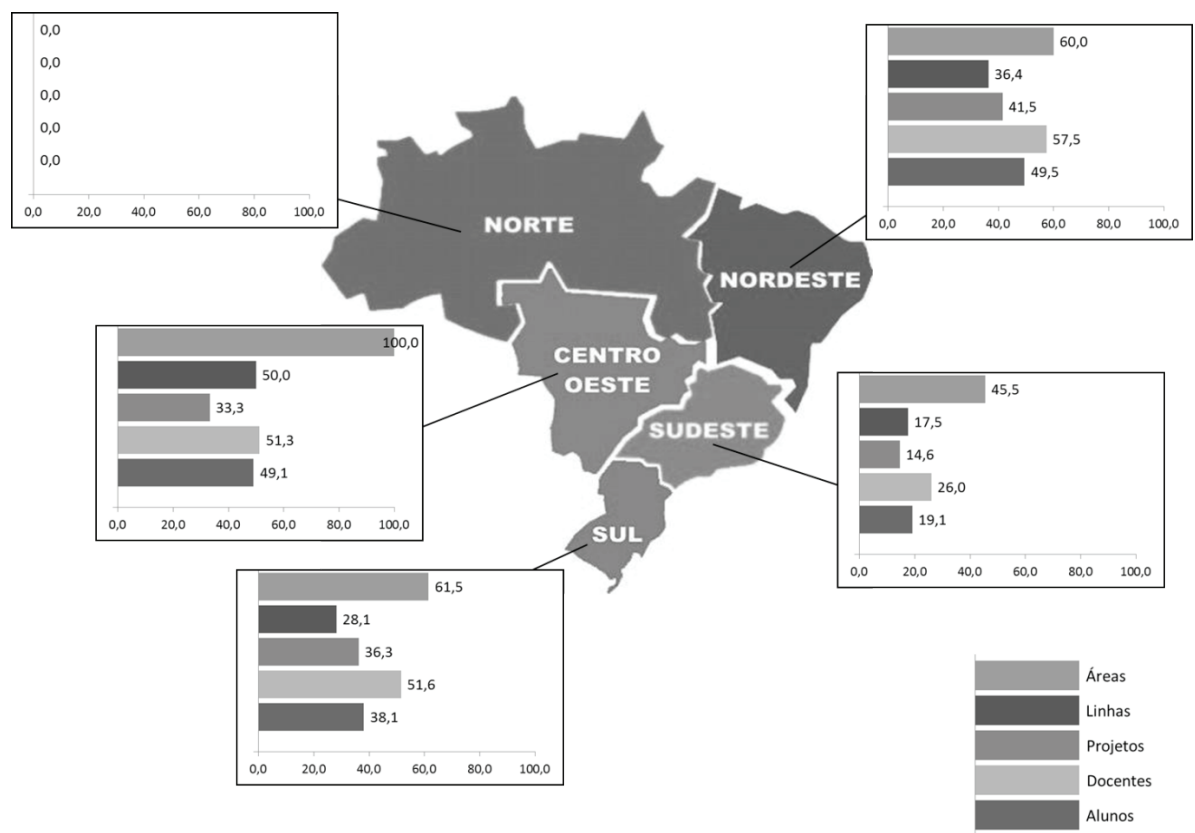

FIGURA 1 - Proporção de áreas de concentração, linhas de pesquisa, projetos de pesquisa em andamento, docentes e alunos vinculados à subárea de Atividade Física e Saúde dentro dos Programas de Pós-Graduação em Educação Física, de acordo com as macrorregiões geográficas, em 2012.

\section{DISCUSSÃO}

A rápida ascensão das doenças não transmissíveis e as transições epidemiológica e nutricional - que representam desafios para a saúde pública em todo o mundo ${ }^{7}$ - têm contribuído para a inclusão da atividade física como estratégia de prevenção, promoção e reabilitação da saúde, assim como para melhoria da qualidade de vida ${ }^{8}$. Nesse contexto, a atividade física vem ganhando espaço em programas e serviços de saúde pública e, consequentemente, o profissional de EF, como um dos atores envolvidos na elaboração e execução dessas ações ${ }^{9}$. Apesar do espaço conquistado dentro da saúde pública, não se deve esquecer que a subárea de AFS não pode perder espaço dentro da área da EF, pois permeia outros espaços de atuação profissional, como o âmbito escolar. Afinal, são os cursos de EF que formam a maior parte dos recursos humanos que atuará em programas de atividade física para a população, além de terem entre os objetos de estudo a atividade física e a forma como ela se relaciona à 
saúde populacional e a outros fenômenos pertinentes à EF.

Nesse cenário, destaca-se a importância da AFS dentro dos PPG em EF. Este estudo identificou que 56\% das áreas de concentração, $25 \%$ das linhas de pesquisa, $26 \%$ dos projetos de pesquisa, $39 \%$ dos docentes e $30 \%$ dos alunos estão relacionados à AFS. Isso reforça o reconhecimento e a valorização da subárea, que nos últimos anos vem crescendo em quantidade e qualidade, apesar de ser relativamente nova. Esse resultado contraria estudo realizado em 2011, no qual as autoras buscaram discutir a heterogeneidade epistemológica dos PPG em EF e a temática "atividade física e saúde" não esteve entre as mais frequentes ${ }^{10}$.

Foi encontrada também uma maior concentração de PPG com conceito 3 em 2012 (50\%), assim como em estudos anteriores ${ }^{11,12}$, demonstrando que a maior parte dos PPG em EF ainda está em processo de qualificação e consolidação. Nesses programas, a subárea AFS apresentou importante inserção, assim como naqueles com conceito 5. A maior inserção da subárea de AFS em PPG criados mais recentemente e ainda em consolidação pode estar associada ao fato de esta ser uma subárea relativamente mais recente do que as outras na EF. Uma possível hipótese de que a subárea de AFS dificulta a consolidação dos PPG perde sustentação quando se observa que a maior inserção está justamente nos PPG com conceito 5. Ademais, dados sobre projetos de pesquisa e bolsistas de produtividade financiados pelo Conselho Nacional de Desenvolvimento Científico e Tecnológico $(\mathrm{CNPq})$, dentro da área de $\mathrm{EF}$, apontam que a proporção de investimentos na subárea de AFS é maior do que em outras subáreas mais tradicionais, indicando que os projetos e pesquisadores relacionados com a AFS têm sido considerados como de boa qualidade e de importante impacto pela agência de fomento e pares ${ }^{13}$.

Em contrapartida, nos dois PPG mais consolidados, com conceito 6 na época, a inserção da subárea é baixa. Isso se deve, provavelmente, por serem PPG mais antigos e historicamente terem enfoque em subáreas mais tradicionais da EF. A política acadêmica brasileira tende a tornar as instituições cada vez mais reconhecidas em âmbito nacional e internacional, por meio do aumento da produtividade científica e intercâmbios institucionais. Sendo assim, acredita-se que ocorra uma melhoria na classificação dos PPG pela CAPES, o que pode contribuir futuramente para uma maior quantidade de programas com conceitos 6 e 7, ao contrário do que é encontrado atualmente.

Os PPG apresentam assimetrias e disparidades regionais, seguindo tendência histórica ainda não superada. Por exemplo, em 2000 a região Norte representava apenas $1,1 \%$ dos alunos de pós-graduação de todo o Brasil, considerando mestrado e doutorado juntos. Nas regiões Sul e Sudeste se concentrava a maioria dos PPG $(17,1 \text { e } 75,1 \% \text {, respectivamente })^{14}$. Evidências apontam que a distribuição dos $\mathrm{PPG}^{11}$ e grupos de pesquisa ${ }^{1}$ na área de $\mathrm{EF}$ e grupos de pesquisa em $\mathrm{AFS}^{15}$ apresenta cenário semelhante. Este estudo encontrou o mesmo para a inserção da subárea de AFS nos PPG de EF. É importante ressaltar que a região Norte não apresenta nenhum PPG em EF e isso pode ocorrer por vários fatores.

$\mathrm{O}$ apoio financeiro recebido pelos órgãos de fomento varia de acordo com diversos critérios, inclusive a produção científica do programa. Sendo assim, é provável que exista um processo cíclico, em que a falta de produção e/ou projetos e grupos de pesquisa influencie a captação de recursos financeiros para 
compra de equipamentos e melhoria de instalações para o desenvolvimento de pesquisa, o que acaba não atraindo professores e/ou alunos para o local. Consequentemente, profissionais de EF acabam não tendo oportunidade de formação continuada na região, e saem em busca de pós-graduação em outras áreas ou em outras regiões do país, principalmente nas regiões Sul e Sudeste. Desta forma, a falta de pesquisadores qualificados na área dificulta a implantação de PPG na região ${ }^{16}$. Evidências mostram que a região Norte apresenta também as menores proporções de grupos de pesquisa ${ }^{1,15}$, o que reforça a importância da pesquisa na implantação de novos PPG e na inserção da subárea de AFS.

Sendo assim, esforços que fomentem a formação de pesquisadores da região Norte na subárea de AFS por outros programas do país e, em seguida, que ofereçam condições para que eles retornem e se fixem na região, podem favorecer a inserção da subárea no interior de novos PPG em EF, além de ocupar espaço destacado em outras áreas das ciências da saúde ${ }^{17}$.

As regiões Centro-Oeste, Nordeste, Sudeste e Sul, em geral, parecem apresentar um panorama positivo quanto à inserção da AFS nos programas da área de EF. Isso pode ser um reflexo do grande crescimento da pesquisa em AFS dentro do Brasil. Nahas e Garcia ${ }^{8}$ atribuem o crescimento da subárea a diversos fatores, entre eles a inclusão da EF como área nas agências de fomento, intercâmbios entre estudantes brasileiros e do exterior, aumento da quantidade e qualidade dos grupos de pesquisa e criação de novos programas.

Entre as limitações deste estudo podemos citar a defasagem temporal dos dados, pois no momento da coleta de dados as informações mais recentes da avaliação de cursos disponibilizada pela CAPES eram de 2012. Atualmente, novos programas surgiram e algumas características podem ter se modificado nesse período. Outro ponto importante foi a dificuldade de definir operacionalmente quais linhas de pesquisa estavam vinculadas à AFS, cuja definição é ampla. Tentou-se superar essa limitação por meio da leitura em pares dos títulos e descrições das linhas de pesquisa, destacando-se que os avaliadores eram pessoas familiarizadas com a subárea. Também se deve atentar que as áreas de concentração podem incorporar linhas de pesquisa que por vezes não refletem o escopo delas. Isso pode acarretar na superestimação de áreas de concentração engajadas com algum tema específico, neste caso a AFS. Por fim, não foi possível definir a inserção da AFS dentro dos PPG em comparação com as demais subáreas da EF, em virtude da heterogeneidade das linhas de pesquisa e da dificuldade de determinar a vinculação com a subárea mais adequada por parte dos avaliadores. No entanto, tal limitação não prejudica severamente a resposta ao objetivo, pois ainda foi possível analisar a inserção da AFS em comparação com o total das demais subáreas.

Conclui-se que a subárea de AFS tem posição de destaque nos PPG em EF no Brasil. No entanto, desequilíbrios regionais e entre instituições mais e menos consolidadas ainda existem em relação à inserção da subárea. Tais fenômenos apontam desafios para que a AFS continue seu processo de amadurecimento no país.

\section{Contribuiccões dos autores}

Todos os autores contribuíram de forma igual no planejamento do estudo, na coleta de dados e na escrita e revisão do manuscrito. 


\section{REFERÊNCIAS}

1. Del Duca GF, Garcia LMT, Silva KS, Nascimento JV. Grupos de pesquisa em cursos de Educação Física com pós-graduação "stricto sensu” no Brasil: análise temporal de 2000 a 2008. Rev Bras Educ Fis Esporte. 2011;25(4):607-17.

2. Kroeff MS, Nahas MV. Ações governamentais e formação de pesquisadores em Educação Física no Brasil. Rev Bras Cienc Esporte. 2003;24(2):114-26.

3. Silva SG, Minatto G, Fares D, Del Duca GF, Nahas MV. Análise temporal de estudos epidemiológicos produzidos em dissertações e teses no Programa de Pós-Graduação em Educação Física da Universidade Federal de Santa Catarina. Rev Bras Ativ Fis Saude. 2012;17(1):22-7.

4. Loch MR. Atividade Física e Saúde nos programas de pós-graduação no Brasil: breve análise a partir de Thomas Kuhn. Rev Bras Ativ Fis Saude. 2012;17(1):46-51.

5. Hallal PC, Dumith SC, Bastos JP, Reichert FF, Siqueira FV, Azevedo MR. Evolução da pesquisa epidemiológica em atividade física no Brasil: revisão sistemática. Rev Saude Publica. 2007;41(3):453-60.

6. Coordenação de Aperfeiçoamento de Pessoal de Nível Superior. Relação de cursos recomendados e reconhecidos [2014 mar 11].Disponível em: http://conteudoweb.capes. gov.br/ conteudoweb/ ProjetoRelacaoCursosServlet?acao=pesquisarIes\&codigoArea= $40900002 \&$ descricaoArea $=\&$ descricao AreaConhecimento EDUCA \% C $7 \%$ C 3 O + F \% CD S CA\&des cricaoArea Avaliaca o = EDUCA\%C7\%C3O+F\%CDSICA.

7. Alleyne G, Binagwaho A, Haines A, Jahan S, Nugent R, Rojhani A, et al. Embedding non-communicable diseases in the post-2015 development agenda. Lancet. 2013;381(9866):566-74.

8. Nahas MV, Garcia LMT. Um pouco de história, desenvolvimentos recentes e perspectivas para a pesquisa em Atividade Física e Saúde no Brasil. Rev Bras Educ Fis Esp. 2010;24(1):135-48.

9. Ministério da Saúde (BR). Diretrizes do NASF: Núcleo de Apoio a Saúde da Família. Brasília: Ministério da Saúde; 2010.

10. Rosa S, Leta J. Tendências atuais da pesquisa brasileira em Educação Física: parte 2: a heterogeneidade epistemológica nos programas de pós-graduação. Rev Bras Educ Fís Esp. 2011;25(1):7-18.

11. Manoel EJ, Carvalho YM. Pós-graduação na Educação Física brasileira: a atração (fatal) para a Biodinâmica. Educ Pesqui. 2011;37(2):389-406.

12. Quadros H, Afonso M, Ribeiro J. O cenário da pós-graduação em Educação Física: contextos e possibilidades na região sul do Brasil. Rev Bras Ativ Fis Saude. 2013;18(5):576-84.

13. Silva ICM, Santin-Medeiros F, Bertapelli F, Coelho APS, Silva SG. Pesquisa em Atividade Física e Saúde no Brasil: um recorte dos investimentos em projetos de pesquisa e bolsas de produtividade CNPq. Rev Bras Ativ Fis Saude. Em avaliação.

14. Balbachevsky E. A pós-graduação no Brasil: novos desafios para uma política bemsucedida. In: Brock C, Schwartzman S, editors. Os desafios da educação no Brasil. Rio de Janeiro: Nova Fronteira; 2005.

15. Santos A, Bastos LLA, Aleixo AA, Paulo TRS, Mendes EL. Distribuição, evolução e produção científica dos grupos de pesquisa em Atividade Física e Saúde do Brasil. Rev Bras Ativ Fis Saude. 2012;17(4):258-62.

16. Kokubun E. A avaliação da Educação Física em debate: esclarecimentos. Rev Bras Pos Grad. 2011;1(2):195-200.

17. Barros MVG. A pesquisa e a formação de pesquisadores em Atividade Física e Saúde no Brasil. Rev Bras Ativ Fis Saude. 2013;17(3):161-2.

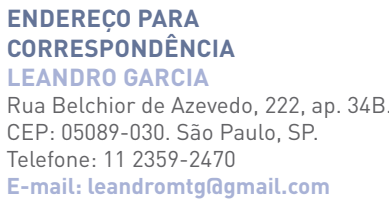

